\title{
Sphenopalatine ganglion block: an external gate to modulate cardiac autonomic tone and suppress premature ventricular beats?
}

\author{
Dimitrios N. Katsaras ${ }^{1}$, Chrysa K. Arvaniti ${ }^{2}$, Panayota G. Flevari ${ }^{1}$, Georgios V. Giannopoulos ${ }^{3}$, Chrysanthi \\ Z. Batistaki ${ }^{4}$, Vassilios E. Stassinos ${ }^{1}$, Georgia G. Kostopanagiotou ${ }^{4}$, Spyridon G. Deftereos ${ }^{1}$, Efstathios K. \\ Iliodromitis ${ }^{1}$, Dionyssios I. Leftheriotis ${ }^{1}$ \\ ${ }^{1}$ Department of Cardiology, ${ }^{2}$ Department of Neurology, “Attikon” University Hospital of Athens, Athens, Greece; ${ }^{3}$ Department of Cardiology, \\ “Georgios Gennimatas" General Hospital of Athens, Athens, Greece; ${ }^{4}$ Department of Anesthesiology, "Attikon” University Hospital of Athens, \\ Athens, Greece \\ Contributions: (I) Conception and design: DI Leftheriotis, CK Arvaniti, DN Katsaras; (II) Administrative support: GG Kostopanagiotou, SG \\ Deftereos, EK Iliodromitis; (III) Provision of study materials or patients: DI Leftheriotis, CK Arvaniti, PG Flevari, CZ Batistaki; (IV) Collection \\ and assembly of data: GV Giannopoulos, DN Katsaras, VE Stassinos; (V) Data analysis and interpretation: GV Giannopoulos, DN Katsaras, VE \\ Stassinos, DI Leftheriotis, CK Arvaniti, PG Flevari; (VI) Manuscript writing: All authors; (VII) Final approval of manuscript: All authors. \\ Correspondence to: Dionyssios I. Leftheriotis, MD. Attikon University Hospital of Athens, Rimini str. 1, Athens, 12462, Greece. \\ Email: dleftheriotis@gmail.com.
}

Background: Autonomic modulation is used for treating various cardiovascular diseases, such as cardiac arrhythmias. Sphenopalatine ganglion (SPG) block is an easy, non-invasive therapy for migraine with a potential cardiovascular impact that remains unclear. In this study, we sought to assess the effect of SPG block on cardiac autonomic tone, as expressed by heart rate variability (HRV), and on ventricular arrhythmogenesis.

Methods: Forty patients (14 male and 26 female) suffering from migraine were randomized by 1:1 to SPG block or placebo (controls) and HRV parameters were evaluated 1 hour before and hourly after the intervention. Twenty-four additional patients (11 men and 13 women) with premature ventricular contractions (PVCs) from the right ventricular outflow tract underwent the same randomization and the number of PVCs was assessed during 1 hour before and every hour after treatment. Values were summarized as median (1st-3rd quartile).

Results: During the first four hours after SPG block, an increase in mean RR [883 (IQR, 869-948) vs. 839 (IQR, 806-887) ms at baseline, $\mathrm{P}<0.01$ ], SDNN [64 (IQR, 59-69) vs. 51 (IQR, 47-55) ms, $\mathrm{P}<0.01$ ], SDANN [39 (IQR, 36-43) vs. 27 (IQR, 22-29) ms, $\mathrm{P}<0.01$ ], ASDNN [51 (IQR, 47-53) vs. 40 (IQR, 37-44) ms, $\mathrm{P}<0.01$ ], rMSSD [30 (IQR, 27-32) vs. 25 (IQR, 23-27) ms, P<0.01], VLF [26 (IQR, 24-29) vs. 23 (IQR, 22-25) $\mathrm{ms}^{2}$, $\mathrm{P}<0.01]$ and $\mathrm{HF}\left[14(\mathrm{IQR}, 11-16)\right.$ vs. $\left.11(\mathrm{IQR}, 9-12) \mathrm{ms}^{2}, \mathrm{P}<0.01\right]$, along with a decrease in LF/HF ratio [1.7 (IQR, 1.4-1.9) vs. 2.0 (IQR, 1.7-2.5), $\mathrm{P}<0.01$ ] was observed in patients with migraine. In patients with PVCs, the number of ectopic ventricular beats per hour was decreased for the first five hours following SPG block [360 (IQR, 264-850) from 956 (IQR, 545-1,412), P<0.001]. No such differences were observed in controls.

Conclusions: SPG block is associated with a transient increase in those HRV parameters that mainly express parasympathetic activity. It is also followed by a significant decrease in ventricular arrhythmic burden. These findings imply an effect on cardiac autonomic tone with a potential favorable clinical impact on arrhythmogenesis.

Keywords: Sphenopalatine ganglion (SPG); autonomic tone; heart rate variability (HRV); premature ventricular beats

Submitted Jun 14, 2018. Accepted for publication Oct 29, 2018.

doi: 10.21037/atm.2018.11.30

View this article at: http://dx.doi.org/10.21037/atm.2018.11.30 


\section{Introduction}

The sphenopalatine ganglion (SPG) is a large extracranial ganglion located in the pterygopalatine fossa near the tail or posterior aspect of the middle nasal turbinate, and contains parasympathetic fibers that synapse within the ganglion, along with sympathetic nerve fibers passing through it (1). Due to the fact that SPG is easily accessed via the naris, SPG lesioning or block has been applied as a non-invasive adjunctive treatment for drug-refractory migraine, cluster headache, and facial pain $(2,3)$. During this technique, a local anesthetic agent or radiofrequency (RF) current can be accurately delivered to the area of mucosa associated with the SPG (3-5).

There are data to suggest that migraine, SPG function and cardiovascular function share common pathophysiological pathways. In patients with migraine, a decreased coronary flow reserve has been reported and a common vasomotor pathway has been proposed as one of the underlying mechanisms (6). Experimental stimulation of the nasal mucosa caused changes in heart rate via afferent pathways involving the trigeminal nerve and the medullary dorsal horns (7). In humans, low frequency SPG stimulation has been associated with increased sympathetic tone and heart rate (8). Recently, a favorable impact of SPG block on systemic blood pressure has been described, indicating a potential effect of this method on the cardiovascular system (9).

Although the facial parasympathetic output of SPG has been systematically studied, a potentially more generalized effect of SPG stimulation or block, including an impact on cardiac function, has not been sufficiently assessed. In the present study, we evaluated the effect of temporary SPG block on cardiac autonomic tone as expressed by heart rate variability (HRV), in patients suffering from migraine. Additionally, the impact of SPG block on the arrhythmic burden was evaluated in patients with idiopathic premature ventricular contractions (PVCs) from the right ventricular outflow tract (RVOT).

\section{Methods}

A total of 64 patients were included in the study. Initially, 40 consecutive patients followed in the Pain Clinic for drug-resistant migraine were divided into an investigational and an observational control group of twenty subjects each, in a random fashion. Randomization took place in a 1:1 consecutive order to transnasal SPG blockade (SPGB group) or placebo-treatment (controls). All patients were symptomfree for at least 48 hours before randomization. They were free of heart disease and arrhythmias which would affect HRV or would necessitate $\beta$-blockers and antiarrhythmic drugs. Patients with other systemic comorbidities (diabetes, renal failure, pulmonary or connective tissue disease, etc.) were also excluded. Initially, a Holter recorder was positioned and $\mathrm{HRV}$ in the time and frequency domain was assessed for one hour before intervention. Subsequently, SPG block was achieved by inserting cotton-tipped swabs soaked in lidocaine hydrochloride $2 \%$ solution to the pterygopalatine fossa through the nasal cannula, as previously described (3). Similarly, normal saline $0.9 \%$ was applied to the SPG in patients randomized to the control group. The application of lidocaine or normal saline lasted twenty minutes, with the patients remaining in supine position. After that, HRV parameters were reassessed every hour and consequently compared to those before the intervention, in order to detect possible changes and to evaluate the duration of SPG block effect. The evaluated parameters in the time domain were the mean of $\mathrm{R}-\mathrm{R}$ intervals (mean $\mathrm{NN}$ ), the standard deviation of the R-R intervals $(\mathrm{SDNN})$, the standard deviation of the averages of 5-minute R-R intervals (SDANN), average of all 5-min SDNN (ASDNN), and the square root of the mean squared differences of successive R-R intervals (rMSSD). In the frequency domain the following parameters were assessed: power in very low frequency range (VLF), power in low frequency range (LF), power in high frequency range $(\mathrm{HF})$, ratio of low to high frequency power (LF/HF), and the total power (whole band, WB).

Additionally, 24 patients (11 men and 13 women)who visited the Arrhythmia Department because of frequent idiopathic PVCs originating from the RVOT underwent the same 1:1 randomization (12 patients in each group) into SPGB (PVC-SPGB group) or controls (PVC-control group), as described above. PVCs were documented in previous Holter recordings or electrocardiograms, and antiarrhythmic medication remained unchanged during the study. The absence of structural heart disease was documented by patients' history, echocardiographic evaluation, exercise stress-test, coronary angiographic, and cardiac magnetic resonance imaging, if indicated. According to the aforementioned schedule, baseline Holter monitoring was performed for one hour before intervention and the number of PVCs per hour was assessed. Following SPG block or placebo-treatment, monitoring was repeated and the number of PVCs was hourly assessed and compared 
Table 1 Demographic characteristics and HRV parameters at baseline in the two groups

\begin{tabular}{|c|c|c|c|}
\hline Characteristics & SPGB $(n=20)$ & Controls $(n=20)$ & $P$ value \\
\hline Female & $12(60 \%)$ & $14(70 \%)$ & 0.7 \\
\hline Hypertension & $10(50 \%)$ & $9(45 \%)$ & 0.8 \\
\hline Hyperlipidemia & $3(15 \%)$ & $4(20 \%)$ & 0.7 \\
\hline Mean NN (ms) & 839 [806-887] & 848 [802-908] & 0.53 \\
\hline SDNN (ms) & $51[47-55]$ & $51[48-55]$ & 0.72 \\
\hline SDANN (ms) & 27 [22-29] & 25 [21-27] & 0.22 \\
\hline ASDNN (ms) & $40[37-44]$ & $42[36-46]$ & 0.67 \\
\hline $\operatorname{LF}\left(\mathrm{ms}^{2}\right)$ & 21 [19-23] & 22 [19-24] & 0.35 \\
\hline $\mathrm{HF}\left(\mathrm{ms}^{2}\right)$ & $11[9-12]$ & 11 [10-12] & 0.23 \\
\hline WB $\left(\mathrm{ms}^{2}\right)$ & 35 [32-36] & 35 [32-36] & 1.00 \\
\hline LF/HF & $2.0[1.7-2.5]$ & $2.0[1.7-2.3]$ & 0.76 \\
\hline
\end{tabular}

HRV, heart rate variability; mean HR, mean heart rate; bpm, beats per minute; mean NN, mean of R-R intervals; ms, milliseconds; SDNN, standard deviation of the R-R intervals; SDANN, standard deviation of the averages of 5-minute R-R intervals; ASDNN, average of all 5-min SDNN; rMSSD, square root of the mean squared differences of successive R-R intervals; VLF, very low frequency range; LF, low frequency range; HF, high frequency range; WB, whole band; LF/HF, ratio of low to high frequency power; SPGB, sphenopalatine ganglion block group.

to baseline. HRV parameters were not assessed, because the presence of frequent ectopic beats is a limitation for the reliable HRV evaluation $(10,11)$.

SEER Light Extend Holter recorders (General Electric, Suzuken Company, Nagoya, Japan) were used for heart rhythm monitoring. All patients and the doctors who analyzed the recordings were unaware to which group (SPGB or controls) each patient belonged. The study protocol was approved by the Hospital Ethics Committee (Eudra CT: 2018-000742-19) in accordance with the Helsinki Declaration as revised in 2013, and all patients gave informed consent before enrollment.

Continuous variables were summarized as median $\left(1^{\text {st }}-3^{\text {rd }}\right.$ quartile $)$ and were compared with non-parametric tests (Wilcoxon and Friedman for two or more than two, respectively, related samples, and Mann-Whitney $U$ test for two independent samples). Categorical variables were summarized as counts and percentages and were compared with Fisher exact test. Two-sided $\mathrm{P}$ values of less than 0.05 were considered as indicating statistical significance.

We calculated that, in order to detect a $5 \%$ difference in mean $\mathrm{NN}$ value (assuming a value of $800 \mathrm{~ms}$ in controls and a standard deviation of $40 \mathrm{~ms}$ ), we needed 17 experimental and 17 control subjects at a power level of 0.80 , with a Type I error probability associated with this test (alpha) of 0.05 . In terms of the pairwise comparison in patients with PVCs, in order to detect a $15 \%$ reduction in the number of PVCs per hour (assuming a baseline value of 1,000), we needed 24 pairs of measurements at a power level of 0.80 , with Type I error probability (alpha) of 0.05 .

\section{Results}

Among patients suffering from migraine, no differences between the SPGB group and the controls were observed regarding the demographic characteristics, comorbidities and baseline HRV parameters (Table 1). Hypertension was treated with calcium channel antagonists, angiotensinconverting enzyme inhibitors or angiotensin II receptor antagonists, while patients with hyperlipidemia were under treatment with statins. Patients under $\beta$-blockers were not found among the studied population, according to the 
inclusion criteria. Besides, $\beta$-blockers are not considered as fist-choice medication in relatively young, physically active patients with uncomplicated hypertension and without inappropriately increased heart rate or stress, according to the guidelines of the European Society of Cardiology (12).

Although no differences in baseline HRV parameters were observed between the two groups, significant deviations developed after SPG block (Table 2), comparison between groups). Following block, a decrease in heart rate and a significant increase in mean NN, SDNN, SDANN, ASDNN, rMSSD, VLF and HF, along with a decrease in LF/HF ratio were initially observed, and gradually returned to baseline levels within 4 hours. Mean NN, SDNN and rMSSD reached maximum increase during the second hour after treatment. LF was mostly decreased during the third and fourth hour after SPG block, while the maximum increase in $\mathrm{HF}$ was observed during the first hour following treatment. As expected, the LF/HF ratio was decreased $(\mathrm{P}<0.001$ within group), mostly during the second hour of follow-up. Systolic and diastolic blood pressure were stable over time, ranging between 136 (IQR, 123-143)/83 (IQR, 74-92) $\mathrm{mmHg}$ and 130 (IQR, 124-143)/81 (IQR, 72-93) $\mathrm{mmHg}$ in the SPGB group and between 136 (IQR, 125-140)/82 (IQR, 77-92) $\mathrm{mmHg}$ and 129 (IQR, 125-137)/79 (IQR, 74-86) mmHg in controls $(\mathrm{P}>0.05$ within and between groups).

Among patients with PVCs, those in the PVC-SPGB group were comparable to $\mathrm{PVC}$-control group regarding demographic characteristics and treatment (Table 3). No significant differences between groups were observed in terms of PVCs per hour before intervention [956 (IQR, $545-1,412$ ) in the PVC-SPGB group and 741 (IQR, 3921,213) in PVC-control group, $\mathrm{P}=0.2]$. The $\mathrm{PVC}$ burden at baseline was $25.0 \%$ (IQR, 15.5-30.7\%) in the PVCSPGB group and $24.8 \%$ (IQR, 14.4-37.1\%) in PVCcontrols $(\mathrm{P}>0.05)$. In the SPGB group, the number of PVCs per hour was decreased to 457 (IQR, 272-977) in the first hour after the ganglion block, 360 (IQR, 264-850) in the second hour, 537 (IQR, 297-767) in the third hour, 769 (IQR, 472-1,206) in the fourth hour and returned to almost baseline levels of 902 (IQR, 471-1,436) in the fifth hour of follow-up, as shown in Figure $1(\mathrm{P}<0.001$, within-group analysis over time). In controls, on the contrary, the number of PVCs remained at 784 (IQR, 572-1,297) in the first hour of follow-up, 722 (IQR, 492-1,140) in the second hour, 775 (IQR, 516-1,275) in the third hour, 728 (IQR, 4571,291 ) in the fourth hour and 727 (IQR, 480-1,097) in the fifth hour ( $\mathrm{P}=0.84$ within group). In the between group analysis, the greatest difference in the number of PVCs between the PVC-SPGB group and the PVC-control group was observed during the second hour of follow-up, but it did not reach statistical significance $(\mathrm{P}=0.8)$.

A non-significant fluctuation in systolic and diastolic blood pressure was observed over time, between 132 (IQR, 120-142)/84 (IQR, 74-92) mmHg and 129 (IQR, 124-136)/80 (IQR, 76-89) $\mathrm{mmHg}$ in the SPGB group and between 131 (IQR, 123-140)/85 (IQR, 76-92) mmHg and 128 (IQR, 126-137)/84 (IQR, 79-89) mmHg in controls ( $P>0.05$ within and between groups).

\section{Discussion}

In this clinical study SPG blockade was evaluated as an easy, non-invasive method to modulate cardiac autonomic tone, as expressed by HRV and the ventricular arrhythmic burden. SPG block was associated with a transient increase in parasympathetic activity and a favorable decrease in PVCs burden. These original preliminary observations hold clinical importance and provide a strong argument for a detailed investigation of the underlying pathophysiologic mechanisms and a potential long-term clinical effect.

Modulation of the autonomic nervous system (ANS) has emerged as an adjunctive clinical tool in many cardiovascular diseases, such as angina pectoris, hypertension and atrial or ventricular arrhythmias $(9,11,13)$. Stimulation of the nasal mucosa has a vasomotor effect on the coronary circulation and an impact on heart rate, implying an interaction between facial and cardiac autonomic innervation (6-8). In accordance to these reports, we observed changes in HRV that suggest an increased parasympathetic tone, possibly due to a predominant suppression of the sympathetic fibers that pass through the SPG $(14,15)$. However, the response to SPG stimulation or blockade is diverse. Increased parasympathetic activity, expressed as bradycardia, has been reported following SPG stimulation in animals, while in humans low-frequency stimulation was followed by increased heart rate, implying increased sympathetic activity $(6,7)$. These seemingly controversial observations remain unexplained and could be attributed to the involvement of more complicated central neural activity through the trigeminal-parasympathetic reflex and the cranial ANS $(1,7,16)$.

The increased sympathetic activity as expressed by HRV parameters before ventricular tachycardias, the circadian variation of RVOT-originating PVCs or tachycardias, and the induction of these arrhythmias by stimulating the 


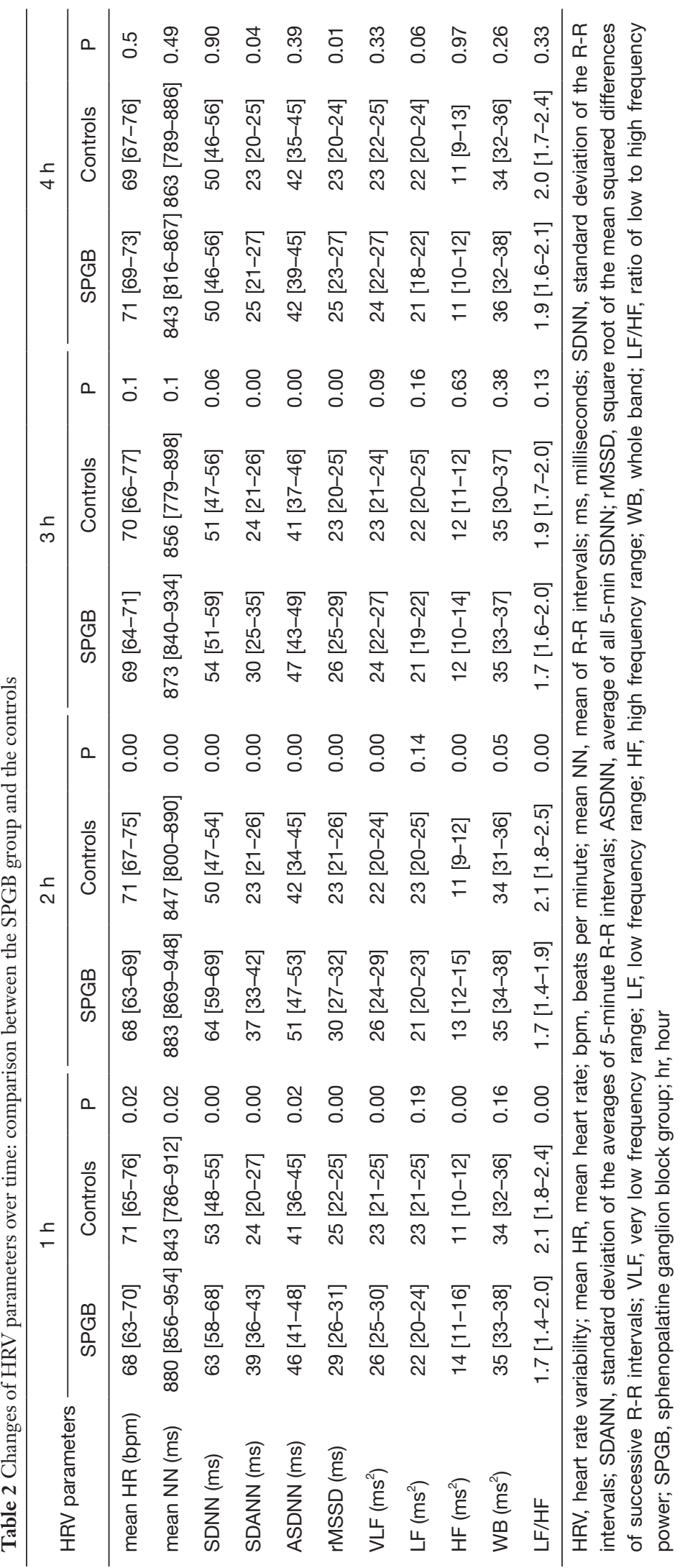


Table 3 Demographic characteristics and treatment in the two groups of patients with PVCs

\begin{tabular}{lccc}
\hline Characteristics & PVC-SPGB group $(\mathrm{n}=12)$ & PVC-control group $(\mathrm{n}=12)$ & $\mathrm{P}$ value \\
\hline Age (years) & $61.0[57.5-67.5]$ & $62.5[56.0-66.5]$ & 0.8 \\
Female & $7(58 \%)$ & $6(50 \%)$ & 1.0 \\
BMI $\left(\mathrm{kg} / \mathrm{m}^{2}\right)$ & $25.8[25.1-26.3]$ & $26.2[25.0-27.6]$ & 0.6 \\
Smoking & $7(58 \%)$ & $8(66 \%)$ & 0.7 \\
Hypertension & $7(58 \%)$ & $6(50 \%)$ & 0.7 \\
Hyperlipidemia & $5(42 \%)$ & $5(42 \%)$ & 0.7 \\
Calcium antagonist & $6(50 \%)$ & $7(58 \%)$ & 1.0 \\
$\beta$-blocker & $6(50 \%)$ & $5(42 \%)$ & 0.7 \\
\hline
\end{tabular}

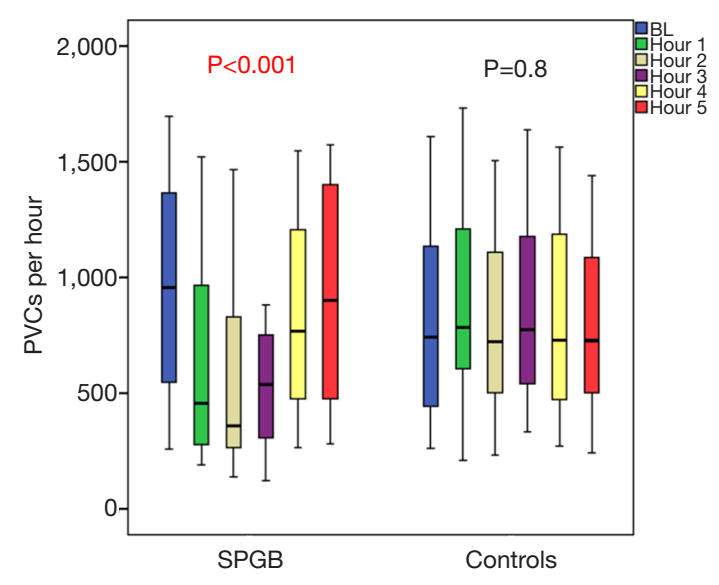

Figure 1 Changes in the number of PCVs per hour over time (within group analysis). PVCs, premature ventricular contractions; SPGB, sphenopalatine ganglion block group. P, P value (maximal difference over time).

sympathetic input to the left pulmonary artery suggest that ANS plays an important role in cardiac electrophysiology and arrhythmogenesis $(17,18)$. The significant decrease in the number of PVCs following SPG block could be attributed to the sympathetic suppression and the predominant parasympathetic activity, which attenuates catecholaminergic stimulation. This effect, combined with the aforementioned changes in HRV, may imply a favorable prognosis in terms of lower arrhythmic risk, as previously assumed (19).

Due to its superficial location, the SPG is easily accessible without the necessity of special devices or needles and might be a gate to cardiac autonomic modulation (20). It is worth mentioning that, in patients with migraine, the analgesic effect of SPG block may last beyond the duration of anesthesia, while a long-term effect of repetitive blockade has been reported (21). Therefore, SPG block could be tested for the acute treatment of ventricular arrhythmias and symptom relief or as an additional tool to reduce the long-term risk of tachycardia-induced cardiomyopathy by repetitive applications, as used in migraine treatment (22). The use of longer than lidocaine acting anesthetics would be a promising alternative to be evaluated.

Despite the original findings of the study, some weaknesses must be taken under consideration. First of all, the study is based on a relatively limited sample size, although the results are significant and provide indications regarding the potential role of SPG block on cardiac autonomic tone. Additionally, the effect of repetitive SPG block, the potential long-term impact of SPG block and its side effects have not been assessed for the present. Furthermore, the pathophysiologic mechanisms involved in this favorable effect remain unclear, and beyond the field of a clinical study like this.

\section{Conclusions}

SPG block is an easy, non-invasive procedure of low cost, associated with an effect on cardiac autonomic tone, expressed by an increase of parasympathetic parameters of HRV. In patients with PVCs from the RVOT, it is associated with a decrease in the arrhythmic burden. These preliminary findings suggest that SPG may represent a potential gateway to autonomic cardiac innervation with antiarrhythmic utility.

\section{Acknowledgements}

None. 


\section{Footnote}

Conflicts of Interest: The authors have no conflicts of interest to declare.

Ethical Statement: The study protocol was approved by the Hospital Ethics Committee (Eudra CT: 2018-00074219) in accordance with the Helsinki Declaration as revised in 2013, and all patients gave informed consent before enrollment.

\section{References}

1. Robbins MS, Robertson C, Kaplan E, et al. The Sphenopalatine Ganglion: Anatomy, Pathophysiology, and Therapeutic Targeting in Headache. Headache 2016;56:240-58.

2. Ivanusic JJ, Kwok MM, Ahn AH, et al.5-HT(1D) receptor immuno-reactivity in the sphenopalatine ganglion: implications for the efficacy of triptans in the treatment of autonomic signs associated with cluster headache. Headache 2011;51:392-402.

3. Maizels $M$, Geiger AM. Intranasal lidocaine for migraine: A randomized trial and open-label follow-up. Headache 1999;39:543-51.

4. Narouze S, Kapural L, Casanova J, et al. Sphenopalatine ganglion radiofrequency ablation for the management of chronic cluster headache. Headache 2009;49:571-7.

5. Yang Y, Oraee S. A novel approach to transnasal sphenopalatine ganglion injection. Pain Physician 2006;9:131-4.

6. Aslan G, Sade LE, Yetis B, et al. Flow in the left anterior descending coronary artery in patients with migraine headache. Am J Cardiol2013;112:1540-4.

7. Hollandsworth MP, DiNovo KM, McCulloch PF. Unmyelinated fibers of the anterior ethmoidal nerve in the rat co-localize with neurons in the medullary dorsal horn and ventrolateral medulla activated by nasal stimulation. Brain Res 2009;1298:131-44.

8. Barloese M, Petersen AS, Guo S, et al. Sphenopalatine ganglion stimulation induces changes in cardiac autonomic regulation in cluster headache. Clin Physiol Funct Imaging 2018;38:808-15.

9. Triantafyllidi H, Arvaniti C, Palaiodimos L, et al. Infiltration of the sphenopalatine ganglion decreases blood pressure in newly diagnosed and never treated patients with essential hypertension. Int J Cardiol 2016;223:345-51
10. Williams B, Mancia G, Spiering W, et al. 2018 ESC/ESH Guidelines for the management of arterial hypertension. Eur Heart J 2018;39:3021-104.

11. Heart rate variability: standards of measurement, physiological interpretation and clinical use. Task Force of the European Society of Cardiology and the North American Society of Pacing and Electrophysiology. Circulation 1996;93:1043-65.

12. Kamath MV, Fallen EL. Correction of heart rate variability signal for ectopics and missing beats. In: Malik M, Cam AJ. editors. Heart Rate Variability. Armonk, NY: Futura 1995;75-86.

13. Ekre O, Eliasson T, Norrsell H, et al. Electrical stimulation versus coronary artery bypass surgery in severe angina pectoris. Long-term effects of spinal cord stimulation and coronary artery bypass grafting on quality of life and survival in the ESBY study. Eur Heart J 2002;23:1938-45

14. Vaseghi M, Barwad P, Malavassi Corrales FJ, et al. Cardiac sympathetic denervation for refractory ventricular arrhythmias. J Am Coll Cardiol 2017;69:3070-80.

15. Chapleau MW, Sabharwal R. Methods of assessing vagus nerve activity and reflexes. Heart Fail Rev 2011;16:109-27.

16. May A, Goadsby PJ. The trigeminovascular system in humans: patho-physiologic implications for primary headache syndromes of the neural influences on the cerebral circulation. J Cereb Blood Flow Metab 1999;19:115-27.

17. Shusterman V, Aysin B, Gottipaty V, et al. Autonomic nervous system activity and the spontaneous initiation of ventricular tachycardia. ESVEM Investigators. Electrophysiologic Study Versus Electrocardiographic Monitoring Trial. J Am Coll Cardiol 1998;32:1891-9.

18. Hasdemir C, Alp A, Aydin M, et al.Human model simulating right ventricular outflow tract tachycardia by high-frequency stimulation in the left pulmonary artery: autonomics and idiopathic ventricular arrhythmias. J Cardiovasc Electrophysiol 2009;20:759-63.

19. Premchand RK, Sharma K, Mittal S, et al. Autonomic regulation therapy via left or right cervical vagus nerve stimulation in patients with chronic heart failure: results of the ANTHEM-HF trial. J Card Fail 2014;20:808-16.

20. Candido KD, Massey ST, Sauer R, et al. A Novel Revision to the Classical Transnasal Topical Sphenopalatine Ganglion Block for the Treatment of Headache and Facial Pain. Pain Physician 2013;16:E769-78.

21. Cady RK, Saper J, Dexter K, et al. Long-term efficacy 
of a double-blind, placebo-controlled, randomized study for repetitive sphenopalatine blockade with bupivacaine vs. saline with the Tx360 device for treatment of chronic migraine. Headache 2015;55:529-42.

22. Hasdemir C, Ulucan C, Yavuzgil O, et al. Tachycardia-

Cite this article as: Katsaras DN, Arvaniti CK, Flevari PG, Giannopoulos GV, Batistaki CZ, Stassinos VE, Kostopanagiotou GG, Deftereos SG, Iliodromitis EK, Leftheriotis DI. Sphenopalatine ganglion block: an external gate to modulate cardiac autonomic tone and suppress premature ventricular beats? Ann Transl Med 2018;6(23):457. doi: 10.21037/ atm.2018.11.30 induced cardio-myopathy in patients with idiopathic ventricular arrhythmias: the incidence, clinical and electrophysiologic characteristics, and the predictors. J Cardiovasc Electrophysiol 2011;22:663-8. 